\title{
Toward Quantitative Liquid Cell Electron Microscopy through Kinetic Control of Solution Chemistry
}

\author{
Mei Wang ${ }^{1}$, Chiwoo Park $^{2}$ and Taylor Woehl ${ }^{1}$
}

${ }^{1 .}$ Department of Chemical and Biomolecular Engineering, University of Maryland, College Park, College Park, USA.

2. Department of Industrial and Manufacturing Engineering, Florida State University, Tallahassee, USA.

Liquid cell electron microscopy (LCEM) enables imaging the structure and dynamics of nanomaterials, energy materials, biological cells, and biomolecules in their near native hydrated state. ${ }^{1}$ Emerging frontiers of LCEM imaging include imaging of soft materials, such as covalent organic frameworks and polymer vesicles, ${ }^{2}$ and biological materials, including DNA-nanocrystal hybrids ${ }^{3}$ and proteins. ${ }^{4}$ Given the propensity of these materials to sustain radiolysis damage, a current outstanding challenge in LCEM imaging is to quantify and mitigate the effects of electron beam induced changes to the sample chemistry. As a corollary, another outstanding challenge is to use the electron beam as a controllable stimulus, a 'quantitative knob,' to actuate or transform nanomaterials through changes in solution chemistry, such a reduction potential or solution $\mathrm{pH}^{5}$

Radiolysis simulations have begun to elucidate the effect of the electron beam on solution chemistry during LCEM imaging. ${ }^{6}$ Effects such as ionic strength increase, $\mathrm{pH}$ change, and redox potential have been identified empirically or by simulation. Each of these effects can impact the structure, chemistry, and dynamics of both hard and soft nanomaterials systems, either by unwanted sample damage or controlled modifications to the sample. The electron dose rate, which is the electron flux divided by the inelastic mean free path of the sample, has been proposed as a 'quantitative knob' for controlling many of these beam induced reactions, both favorable and undesirable.

Prior investigations have suggested that dose rate alone controls the concentration of radical species formed during LCEM imaging, controlling kinetics of processes such as nanocrystal nucleation and growth. Here we present kinetically controlled electron beam induced silver nanocrystal formation experiments that demonstrate dose rate is a much more complex quantitative knob than previously thought. We performed electron beam induced silver nanocrystal formation experiments with LCEM and scanning transmission electron microscopy (STEM) in the presence of an oxidizing radical scavenger to investigate the effects of dose rate on nucleation and growth rates (Figure 1). We found that use of a radical scavenger enables unprecedented repeatability in our experiments and allowed us to collect large data sets to search for new correlations between nanocrystal formation kinetics and dose rate. Surprisingly, neither nucleation nor growth correlated directly with dose rate. Instead we found that growth rate correlates with the electron flux normalized to the density of radiolysis spurs formed in the liquid. Nucleation rate showed no correlation with dose rate, but instead correlated well with the STEM magnification. We rationalized this correlation in terms of the electron beam velocity, which decreases at higher magnifications and enables supersaturation conditions to be established more rapidly. These results indicate that specific details of radiation chemistry must be considered in order to utilize dose rate as a quantitative knob for stimulating reactions during LCEM. Further, these results indicate that nucleation and growth of nanocrystals can be independently controlled with STEM imaging, opening opportunities for studying these phenomena independent of each other with LCEM. 


\section{References:}

[1] N de Jonge and FM Ross, Nature Nanotechnology 6 (11) (2011), p. 695.

[2] LR Parent et al, Accounts of Chemical Research 51 (1) (2018), p. 3.

[3] S Keskin et al, Journal of Physical Chemistry Letters 6 (22)(2015), p. 4487.

[4] D Peckys, BU Korf and N de Jonge, Science Advances 1(6) (2015).

[5] TJ Woehl and P Abellan, Journal of Microscopy 265 (2) (2017), p. 135.

[6] NM Schneider et al, Journal of Physical Chemistry C 118 (38) (2014), p. 22373.
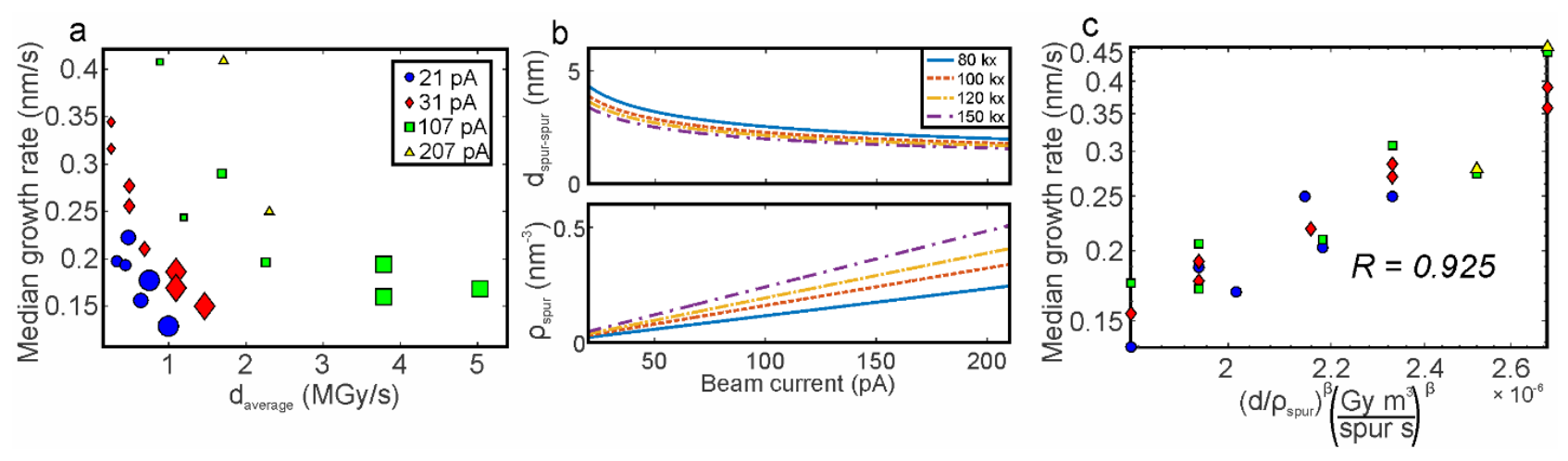

Figure 1. (a) Mean growth rate of silver nanocrystals as a function of viewing area averaged STEM dose rate. Different colors/shapes correspond to constant beam current, while different sized data points indicate the magnification (smallest size, $M=80,000 \mathrm{x}$, largest size, $M=150,000$ ). (b) Spurspur center distance (top) and spur density (bottom) as a function of magnification and beam current. (c) Correlation of mean growth rate with dose rate normalized to spur density, raised to the power of $\beta=0.445$. The value of $\beta$ is the power law dependence of reducing radical concentration on dose rate, determined from radiolysis simulations. 